\title{
Biologie versus Kultur, Sprache und Denken - am Beispiel von Farben und Emotionen
}

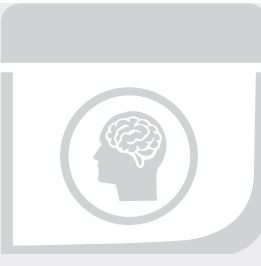

Seit ca. 2 Millionen Jahren sammelten sich Menschen um Feuer, weil es wärmte, Fressfeinde und Insekten vertrieb und später half, die eigene Nahrung durch Erhitzen besser verdauen zu können. ${ }^{1}$ Dadurch konnten sich unsere Vorfahren im weiteren Verlauf ihrer Evolution einen kürzeren Darm leisten, als man bei reiner Rohkost brauchen würde. Dies wiederum ermöglichte ihnen alternative Infrastrukturinvestitionen, nämlich in ein größeres Gehirn, das wiederum zum Erlernen der richtigen Zubereitung von Nahrung immer wichtiger wurde. ${ }^{2}$

Auf Feuer muss man aufpassen, sonst geht es aus. Erst das größere Gehirn erlaubte entsprechende Planung, wegen der Sorge um die Zukunft (nichts zu Essen und zu-

1 Diese Überlegungen setzen sich erst während der letzten Dekade wirklich durch, nahm man früher doch ein jüngeres Zeitalter für die menschliche Nutzung des Feuers an [1]. In einer jüngeren wichtigen Zusammenfassung kann man hierzu Folgendes lessen: "The most obvious aspect of human biology associated with the requirement for a cooked diet is a reduced digestive system compared to great apes, including small molars, mouth, stomach and large intestine. The benefits of gut minimisation include less energy having to be spent in metabolic activity. Since these benefits could not have been realised until high-quality foods were available all year, tooth and gut reduction appear to be unambiguous markers of adaptation to cooking. Furthermore, the calorific benefits of cooked food are so large, the food-softening effect of cooking so strong, and the typical hunter-gatherer diet so difficult to ingest and digest in its raw forms that the adoption of cooking is expected to have a greater influence on tooth and gut reduction than any other dietary factors" [6].

2 Mit den Worten des Anthropologen Joseph Henrich in seinem sehr lesenswerten Buch The Secret of Our Success: "Our bodies, and in this case our digestive system, have coevolved with culturally transmitted knowhow related to food processing “ [8]. „The energy savings from the externalization of digestive functions by cultural evolution became one component in a suite of adjustments that permitted our species to build and run bigger and bigger brains" (S.69). gleich höhere Gefahr, gefressen zu werden), und - besser noch - das Erfinden und die Weitergabe (durch Lehren und Lernen) der Kunst des Feuermachens. Dies erzeugte einen neuen Selektionsdruck für Gehirne: Je mehr es zu lernen und zu speichern gab, desto besser sollten die Gehirne dies auch können. Die Entwicklung von Sprache und Kultur, von Erfahrungswissen für das Jagen, Sammeln, Zubereiten und Haltbarmachen von Nahrung und die Weitergabe dieses Wissens, das so groß ist, dass ein Einzelner nicht mehr alles wissen kann, trieb also die Evolution unseres Gehirns und unserer sozialen kooperativen Fähigkeiten an. Man spricht von einer kumulativen, kulturell getriebenen Evolution des menschlichen Gehirns [14], die nach Art einer Feed-forward-Spirale (also durch positive Rückkoppelung) funktionierte: Größere Gehirne ermöglichten mehr Kultur und mehr Kultur wiederum trieb die Selektion größerer Gehirne an.

Waren Gedanken („-Spiele“) dieser Art noch vor wenigen Jahren nichts weiter als auf alten Knochen und Steinen basierende Gedankenspiele (die damals das Ergebnis hatten, dass der Mensch erst Produkt der Evolution war und dann die Kultur allen weiteren, viel rascheren, Fortschritt verursachte), sind heute aus ihnen empirisch überprüfba- re Hypothesen geworden. Seit den ersten klaren und eindeutigen Beispielen für den Einfluss von Kultur auf unsere Gene (Milchverdauung ${ }^{3}$, blaue Augen ${ }^{4}$ ), hat es geradezu eine Explosion von Studien gegeben, die sich mit den Effekten von Kultur auf die Evolution von Mensch und Tier beschäftigen oder die Wechselwirkungen von kultureller und biologischer Evolution beschreiben. Besonders erwähnenswert erscheint mir eine Arbeit, die aus Überlegungen zu Metabolismus und Energieverbrauch verschiedenen Ursachen (,Treibern“) der Evolution des menschlichen Gehirns nachging

3 Hierzu darf das Gen, das ein Ferment produziert, welches Milchzucker spaltet, nicht - wie vor der Zeit von Ackerbau und Viehzucht - nach dem Abstillen abgeschaltet werden. Eine Mutation des menschlichen Erbguts, die dieses Abschalten verhindert, setzte sich daher dort durch, wo man mit dem Verzehr von Milch begonnen hatte und bis heute gibt es umso weniger Fälle von Laktoseintoleranz in den Gebieten, wo Viehzucht und Milchkonsum schon seit Jahrtausenden praktiziert wird [8].

4 Der Ackerbau bis nördlich des Polarkreises war und ist bis heute nur an der Ostsee möglich, weswegen dort die Vitamin-D-Produktion in der Haut, sofern man nicht Fisch aß, sondern Getreide, besonders bedeutsam und damit auch Mutationen für hellere Haut (und blaue Augen) sich durchsetzen konnten - weltweit einmalig [19].

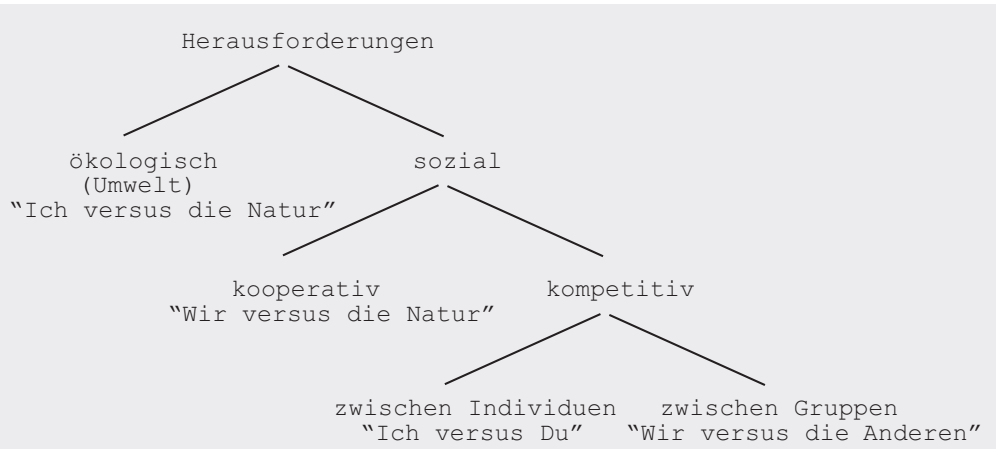

- Abb. 1 Die Triebkräfte der Evolution der Größe des menschlichen Gehirns lassen sich als „Herausforderungen“ verstehen, auf welche die Evolution reagierte. Diese betreffen die Umwelt (ökologisch) und die soziale Umwelt, das herausgeforderte Subjekt ist entweder ein einzelner Organismus oder eine Gruppe, und im Hinblick auf die soziale Umwelt kann es um Konkurrenz oder um Kooperation gehen (nach Daten aus [5], S. 554, Fig. 1). 
(- Abb. 1). Das recht unerwartete Ergebnis: Die Kombination von $60 \%$ Auseinandersetzung mit der Umwelt, $30 \%$ Kooperation und $10 \%$ Gruppenkonflikt trieben die Entwicklung der Größe unseres Gehirns; Konflikte und Konkurrenz zwischen Individuen (gar) nicht ${ }^{5}[5]$.

Mit all dem ist eines klar: Menschen, ihr Denken und Handeln, ist von Biologie und von Kultur bestimmt. Nehmen wir das Beispiel Feuer: Wir verbrennen uns die Finger am Feuer und ziehen die Hand reflexhaft zurück (Biologie). Und wir wissen um Feuer und dessen lebenswichtige Funktionen für uns Menschen (Kultur). Welche Bedeutung hat nun der Begriff Feuer wirklich? Oder anders gefragt: Ist die Bedeutung des Begriffs Feuer eher abhängig von unserer Natur (Biologie) oder eher von unserer Kultur? - Diese sehr grundlegende Frage ist seit längerer Zeit Gegenstand der Sprachwissenschaft. Bei Automarken oder Steinen ist es recht leicht, Kultur und Natur als Quelle der Bedeutung auszumachen. Aber wie ist es bei Hunden und Apfelbäumen? Hier waren es zweifellos Natur und Kultur (Zucht) am Werk.

Menschen reden über die Dinge und über sich selber, in mehr als 6000 unterschiedlichen Sprachen. Deren Wörter sind sehr unterschiedlich. Ist das, was die Wörter meinen, also die Bedeutung der Wörter, auch so unterschiedlich? - Die Frage ist leicht gestellt, aber lässt sie sich wirklich beantworten? - „Fragen Sie doch einfach die Leute!“, könnte man hier bemerken, wären damit nicht die gleichen Probleme, nur eben noch einmal, angesprochen: Wie kann man reden, wenn man gar nicht sicher ist, ob man das Gleiche meint und denkt? Aber denken alle Menschen gleich? - Wieder eine ganz einfache Frage, die umso schwieriger wird, je länger man darüber nachdenkt.

Man kann versuchen, diese Fragen dadurch zu beantworten, dass man die Bedeutungen von Wörtern genauer untersucht. „Bedeutung ist notorisch schwer zu messen und noch schwerer zu parametrisieren, um

5 Wenn man bedenkt, dass man unter „Evolution“ lange den „Kampf ums Dasein“ verstanden hat, und diesen dann auch noch als Kampf einzelner Individuen untereinander (miss-)verstanden hat, ist die Bedeutung dieser Einsichten kaum zu überschätzen! vergleichende quantitative Untersuchungen durchzuführen “6 [29], schreiben Wissenschaftler, die sich dieses Problems angenommen haben, zu Recht. Sie untersuchten die Bedeutung von Wörtern, um herauszufinden, ob Menschen überall begrifflich ähnlich denken oder ob die Struktur ihres Denkens letztlich aus unterschiedlichen Lebensumständen, historischen Zufällen und den Unterschieden der jeweiligen Sprache resultiert. Hierzu machten die Autoren sich die Tatsache zu Nutze, dass Wörter in verschiedenen Sprachen unterschiedliche „Bedeutungshöfe“ haben, d. h. zwar im Wesentlichen das Gleiche meinen, jedoch noch zusätzlich andere Bedeutungsgehalte mitmeinen können. Man spricht von Polysemie (Mehrdeutigkeit), wenn Wörter einer Sprache neben einer „Hauptbedeutung“ noch weitere „Zusatzbedeutungen“ haben.

Polysemie ist häufiger als man denkt. So meint beispielsweise „Bank“ nicht nur ein Möbelstück oder Geldinstitut, sondern im Hinblick auf das Geldinstitut sowohl dessen Funktionsweise als auch das Gebäude, indem diese Funktionen ablaufen. Man macht sich dies jedoch selten klar. Auch das Wort „Läufer“ bezeichnet keineswegs nur einen Menschen, der eine Laufsportart ausübt, sondern auch eine Figur im Schachspiel oder ein junges Schwein, das kein „Baby“ (Ferkel) mehr ist, aber auch noch nicht ausgewachsen. „Läufer“ kann auch einen schmalen, langen Teppich, den oberen sich drehenden Mühlstein, einen längs zur Mauerrichtung verbauten Stein im Mauerwerk, einen die Malfläche herablaufenden Tropfen Farbe, den beweglichen Teil beim Rechenschieber meinen oder noch 16 weitere zum Teil ähnliche, aber teilweise auch völlig andere Dinge/Sachverhalte/Phänomene. ${ }^{7}$ „Flügel“ kann ein Musikinstrument oder einen Körperteil von Vögeln, aber auch einen Teil von einem Gebäude oder eine Seite eines Spielfeldes (Schach, Fußball) bezeichnen. Das Beispiel zeigt sehr schön, dass die Bedeutungen polysemer Wörter in unterschiedlicher Weise verwandt sein können.

6 Im Original: „Meaning is notoriously difficult to measure, let alone parameterize, for quantitative comparative studies.“

7 „Läufer“ ist nach dem Guinness Buch der Rekorde das deutsche Wort mit der höchsten Anzahl - 24 - an Bedeutungen.
Um Polysemie und damit das Wesen von Sprache selbst zu untersuchen, erstellte der US-amerikanische Sprachwissenschaftler Morris Swadesh seit den 1950er-Jahren Wortlisten von wichtigen, möglichst allgemeinen, häufigen und (über längere Zeiträume) stabilen Wörtern - dem basic core vocabulary [23, 24]. Er tat dies in vielen Sprachen, um diese Sprachen dann unter Zuhilfenahme der Listen im Hinblick auf ihre Entstehungsgeschichte zu untersuchen ( Abb. 2).

Eine internationale Arbeitsgruppe um die koreanische Physikerin Hyejin Youn [29] verwendete 22 Wörter aus den Swadesh-Listen, die in 81 Sprachen vorkommende Universalien bezeichnen: Materielle Dinge/Phänomene (Stein, Erde, Sand, Staub, Salz, Feuer, Rauch, Asche, Wasser, Wind), Himmelsobjekte und Zeit-Marker (Sonne, Mond, Stern, Jahr, Tag, Nacht) sowie geografische Umgebungen (Berg, See, Himmel, Wolken, Meer, Fluss [29]).

Durch die Analyse von „Zusatzbedeutungen“ erstellten die Autoren für die 22 Wörter sogenannte „Kolexifikations“-Netzwerke. Wie man sich die vorzustellen hat illustriert $>$ Abb. 3. Dort sind unten in der - Tab. 6 Wörter mit allgemeiner, auf Pflanzen bezogenen Bedeutung - Wald, Baum, Holz, Stamm, Ast und Wurzel - in 5 Sprachen (jeweils in Lautschrift geschrieben) aufgeführt, wobei in bunter Lautschrift diejenigen Wörter angeführt sind, die gleich lauten und damit 2 Bedeutungen aufweisen. Wie also beispielsweise aus der ersten Zeile der Tabelle hervorgeht, gibt es im Französischen ein Wort für Holz („bwa“), das auch „Wald“ bedeuten kann. Im Russischen (Zeile 2) wird für „Baum“ und für „Holz“ das gleiche Wort („djerrva“) verwendet, im Kroatischen (Zeile 3) gibt es nur ein Wort für die Begriffe „Baum“ und „Stamm“ und in 2 weiteren (für den Autor unaussprechlichen) Sprachen gibt es jeweils ein gemeinsames Wort für „Baum“ und „Holz“ (gelb) sowie für „Stamm“ und „Ast“ (grün) (im Yukaghirischen) sowie für „Wald“ und „Baum“ (dunkelblau) und für „Stamm“ und Wurzel (orange) (in Yaqui). Ordnet man dann die Bezeichnungen räumlich an und verbindet sie mit Strichen, deren Dicke die Anzahl der Sprachen, in denen sie eine gleiche Bedeutung haben, entspricht, entsteht ein Kolexifikations-Netzwerk. Was bei einer 
entsprechenden Analyse der 22 Wörter in 81 Sprachen herauskam, zeigt $>$ Abb. 4.

Was kann man mit so etwas anfangen? Eine US-Arbeitsgruppe [10] beantwortet diese Frage so: „Diese Kolexifikations-Netzwerke können interessante Unterschiede in der Bedeutungsstruktur anzeigen, da sie darüber Auskunft geben, wie verschiedene Sprachgruppen die mit Begriffen verbundenen Bedeutungen unterschiedlich handhaben. Wenn beispielsweise alle Sprachen ,Getränk' mit den Begriffen ,Regen‘, ,Fluss` und ,See' in Verbindung bringen, legt dies nahe, dass die meisten Menschen den Begriff, Getränk' mit ,Wasser in der Natur' in Verbindung bringen und damit auf einer tieferen Ebene ,Wasser' als grundlegend für die Bedeutung von ,Getränk' erachten. " Dem wäre nach Meinung der Autoren nicht so, wenn dies nur in einer Sprachgruppe der Fall wäre und eine andere beispielsweise ,Getränk' mit ,süß', ,Frucht' aber auch mit ,See “ und ,Fluss ' in Verbindung bringen würde. Solche unterschiedlichen Muster der Kolexifikation in Sprachgruppen könnten damit Unterschiede in den Bedeutungshöfen der von den Sprachen verwendeten Begriffe anzeigen. ${ }^{8}$ Und sie könnten die Frage klären helfen, ob die

8 Das ganze Argument mit den Worten der Autoren: "These colexification networks can be an insightful indicator of variation in semantic structure because they give information about how different language groups view the associative meaning of concepts. For example, if all spoken languages colexify the concept of "drink" with the concept of "rain," "river," and "lake," this suggests at a basic level that most humans associate the meaning of "drink" with bodies of water, and at a deeper level that most humans view "water" as fundamental to the meaning of "drink." On the other hand, if language group A colexifies "drink" with "lake" and "river" but language group B colexifies "drink" with "sweet," "fruit," and "sugar" as well as "lake" and "river," this would suggest that language group A defines "drink" primarily as water-based whereas language group B defines "drink" as inclusive of a set of concepts involving fruit juice as well as water. Comparing networks of colexification requires some assumed equivalence in concept identity (“drink") across language families, but varying colexification patterns can illustrate how these concepts can have different meanings across the world's spoken languages" [10].
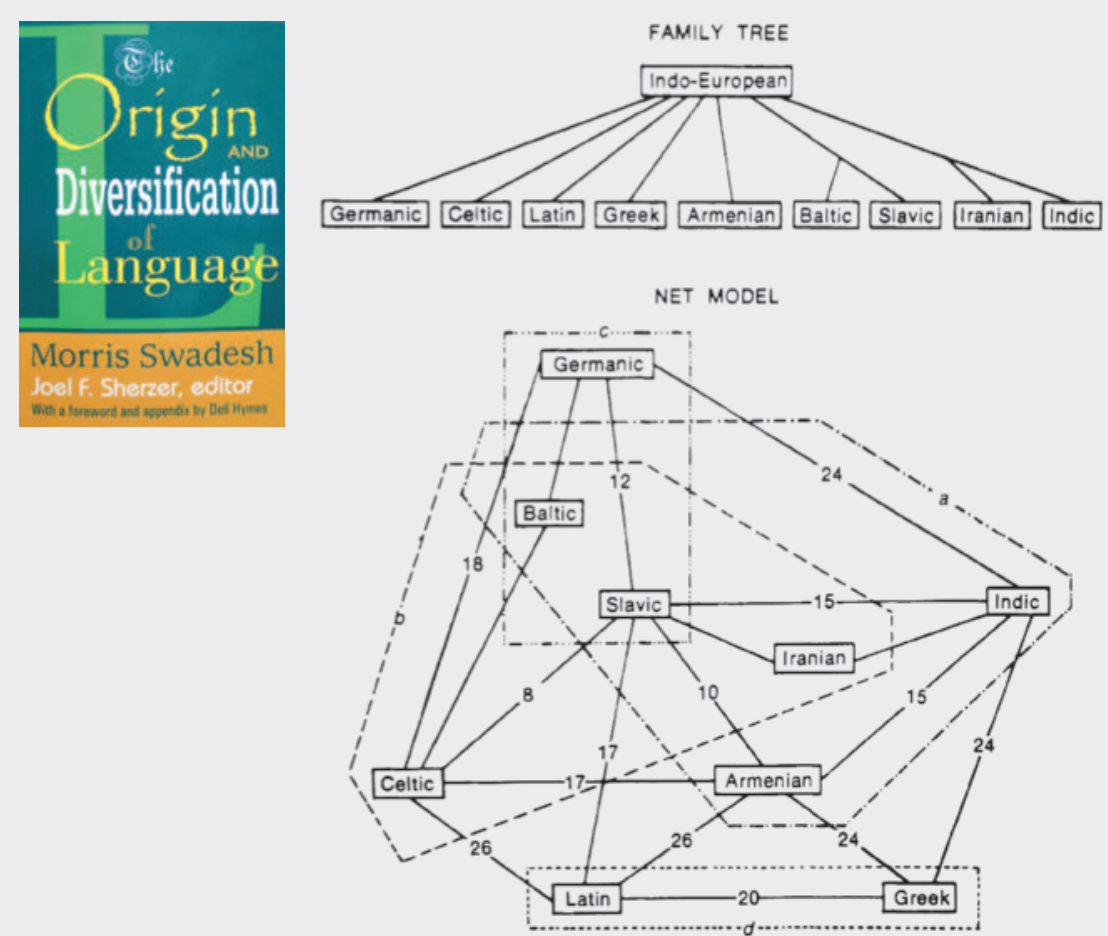

Numbers indicate minimal centuries of divergence, given by glottochronological counts.

Letters indicate some ancient isoglosses:

a: $k>s h$

$b: d h>d$

c: case endings with $m$

d: feminine nouns with masculine suffixes

- Abb. 2 In dem posthum erschienenen Buch (links) des US-amerikanischen Sprachwissenschaftlers Morris Swadesh (1909-1967) findet sich die rechts dargestellte Grafik des Indo-Europäischen Sprachnetzwerks, das Swadesh aufgrund phonologischer Wortähnlichkeiten erstellte. Er argumentierte damals für eine Darstellung als Netz, das ihm zufolge besser zur Darstellung der Struktur von Sprachen geeignet sei als die häufiger verwendeten (Stamm-) Bäume [24].

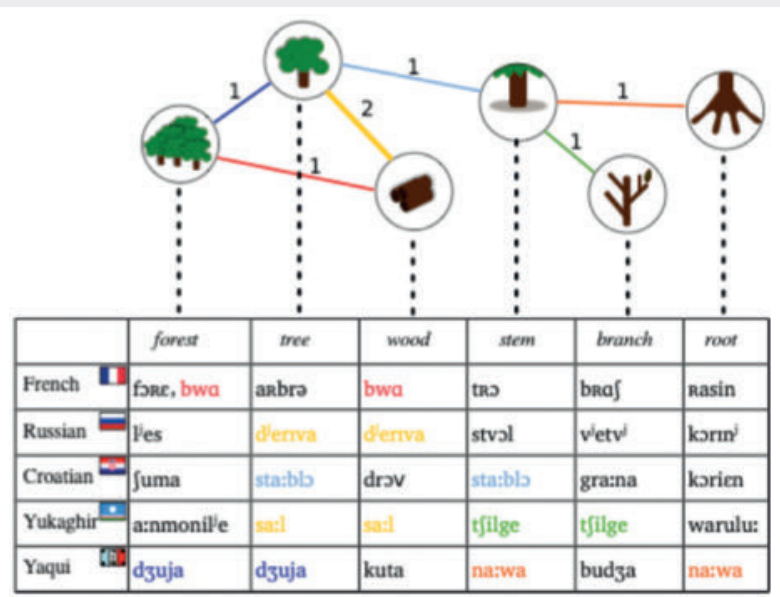

- Abb. 3 Beispiel der Entstehung eines Kolexifikations-Netzwerks der allgemeinen pflanzenbezogenen Begriffe „Wald“, „Baum“, „Holz“, „Stamm“, „Ast“ und „Wurzel“. Die jeweiligen Wörter haben in manchen Sprachen eine doppelte Bedeutung, wie die Tabelle unten mit Hilfe jeweils farbiger Lautschrift anzeigt. Im Netzwerk oben ist die Verbindung von „Baum“ und „Holz“ dicker eingezeichnet, weil in 2 Sprachen für beide Begriffe das gleiche Wort verwendet wird. Alle anderen Verbindungen sind dünn gezeichnet, weil Wortgleichheit für diese Begriffe jeweils nur in einer Sprache vorkommt (nach Daten aus [10]). 


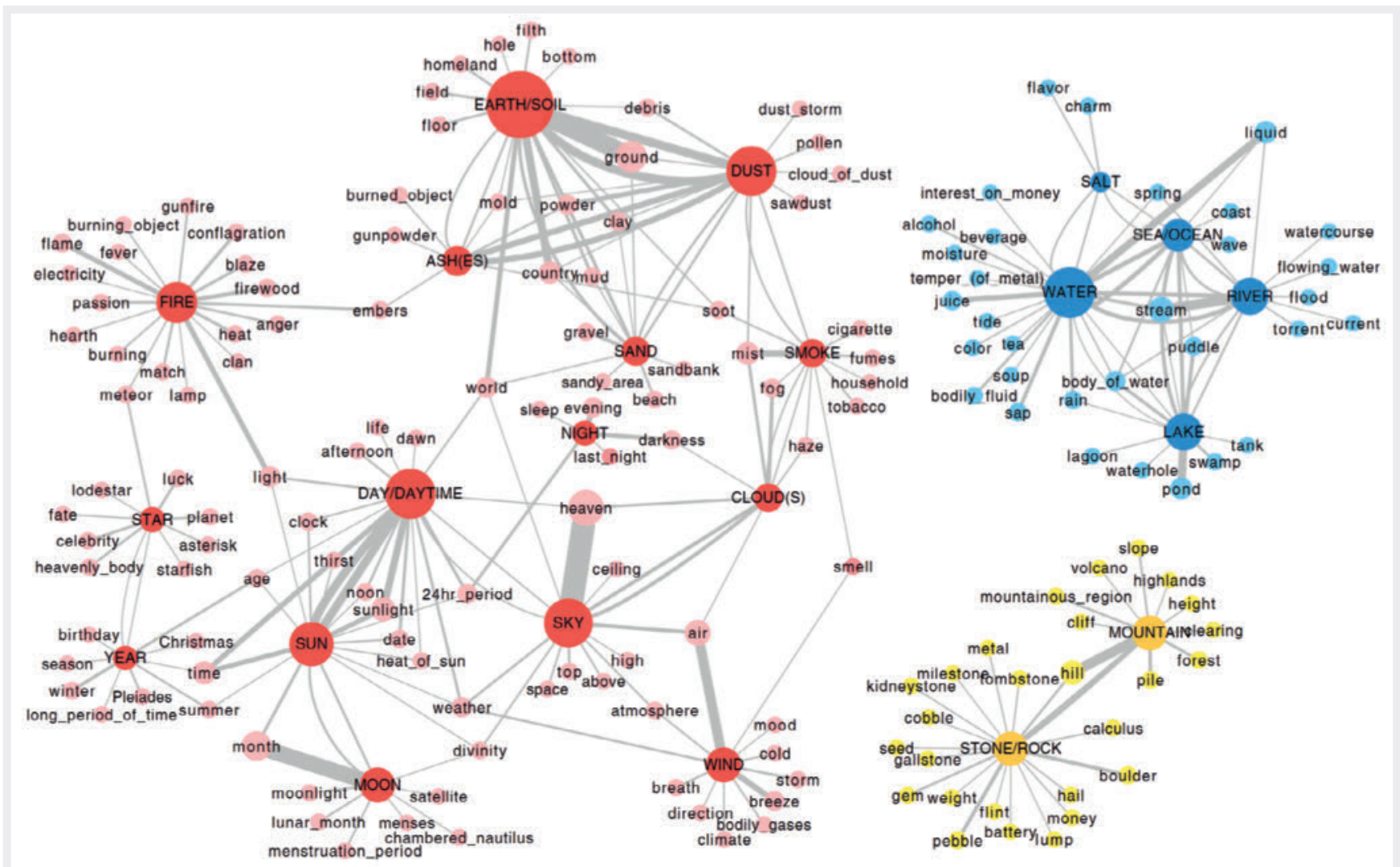

Abb. 4 Kolexifikations-Netzwerk der 22 Wörter (in Großbuchstaben) aus 81 Sprachen, das die Autoren auch als semantisches Netzwerk bezeichnen, da hier Zusatzbedeutungen (in Kleinbuchstaben) von grundlegenden und häufigen Wörtern quantitativ ausgewertet wurden (nach Daten aus [29]). Knoten sind mit Linien verbunden, wenn es Wörter gibt, die beides meinen. Die Größe der Knoten und die Breite der Verbindungen entsprechen der Anzahl der in den 81 Sprachen gefundenen Mehrdeutigkeiten (Polysemie). Zur besseren Übersicht wurden Verbindungen nur dann (durch einen sehr dünnen Strich) eingezeichnet, wenn mindestens 2 Mehrdeutigkeiten vorlagen; auch die Richtung der Verbindungen wurde nicht dargestellt. Wie man sieht, ergab die Analyse 3 getrennte Bedeutungs-Cluster (gelb, rot, blau).

Bedeutung der Begriffe eher von der Natur oder der Kultur bestimmt wird.

So ergab die Analyse der 22 angeführten Wörter, dass deren Bedeutung erstaunlich universell ist. Anders gesagt, die jeweilige Kultur hat einen relativ geringen Einfluss auf Nuancen der Bedeutung dieser Wörter. Dies zeigte sich auch daran, dass die gefundenen geringen Unterschiede keinen Zusammenhang mit geografischen Variablen (z. B. dem Abstand der Sprachen auf dem Erdball) oder kulturellen Variablen wie der Umgebung (feucht, trocken oder kalt) oder dem Vorhandensein von Schrift aufwiesen. Sie beenden die Zusammenfassung ihrer Arbeit mit dem Statement: „Die hier vorgestellte Methode kann auf jegliche Bereiche semantischer Gehalte angewandt werden, um herauszufinden, in welchem Ausmaß die untersuchten begrifflichen Strukturen, in gleicher Weise, universelle Gültigkeit für das menschliche Denken und die Verwen- dung von Sprache haben“9 [29] - „unabhängig von deren Kultur oder Geografie“, ergänzen sie später.

Und genau das taten die Autoren einer internationalen Arbeitsgruppe [10], denn sie wendeten die Methode der Analyse von Polysemie über die Kolexifikation in Sprachfamilien auf menschliche Emotionen an. An-

9 "The methods developed here can be applied to any semantic domain to reveal the extent to which its conceptual structure is, similarly, a universal attribute of human cognition and language use". Und auf der gleichen Seite ergänzen sie etwas später: “Using cross-linguistic dictionaries across languages carefully selected as an unbiased sample reflecting the diversity of human languages, we provide an empirical measure of semantic relatedness between concepts. Our analysis uncovers a universal structure underlying the sampled vocabulary across language groups independent of their phylogenetic relations, their speakers' culture, and geographic environment." statt also Menschen zu ihren Gefühlen zu befragen oder die Reaktionen von Versuchspersonen auf einzelne Reize - Mimik, Gestik, Vokalisationen - zu untersuchen, untersuchten die Autoren der vorliegenden im Fachblatt Science publizierten Studie 2474 (!) Sprachen. „Wenn man analysiert, wie die Leute Wörter benutzen, kann das zeigen, ob die Leute Emotionen ähnlich oder unterschiedlich erfahren “, beschreiben die Autoren ihr Vorgehen“10 [10].

Der Hintergrund ihrer Fragestellung ist nach den dargestellten Gedanken und Erkenntnissen einfach und rasch darzustellen: Wenn wir über Bäume und Flüsse reden, ist klar, worum es geht, denn es gibt sie überall und wir können mit dem Finger darauf zeigen, wenn es Verständigungsprobleme gibt. Wenn wir aber über unsere Gefühle

10 “[...] analyzing how people use words can reveal how they experience emotions as similar or different." 
reden, ist das anders: Woher weiß ich, dass Deine Traurigkeit sich genau so anfühlt wie meine, Deine Wut so wie meine oder Deine Eifersucht so wie meine? Sofern wir die gleiche Sprache sprechen, mag eine Verständigung hier noch halbwegs gelingen, was aber geschieht, wenn wir unterschiedliche Sprachen sprechen?

Gefühle sind einerseits biologisch verankert, andererseits jedoch mehr als „nur“ Biologie. Dies kann man beispielsweise daran festmachen, dass bestimmte Wörter, die Gefühle bezeichnen, schwer zu übersetzen sind. Für „Sehnsucht“ gibt es kein englisches Wort, und manche Wörter bezeichnen eine Emotion und eine zweite Emotion, in einer anderen Sprache jedoch eine Emotion und eine andere, dritte Emotion. Da solche „Zusatzbedeutungen“ letztlich mit der Methode der Kolexifikation erfasst und quantifiziert werden, sollte es möglich sein, mit ihr der begrifflichen Struktur der Emotionen auf die Spur zu kommen. Jackson und Mitarbeiter beziehen sich dabei ausdrücklich auf die 3 Jahre zuvor schon von Youn und Mitarbeitern [29] publizierte Studie. ${ }^{11}$

In den 2474 Sprachen identifizierten sie 2439 Begriffe, von denen 24 Emotionen benennen, die besonders unter die Lupe genommen wurden. Statistisch wurden dann für alle Sprachen Kolexifikations-Netzwerke erstellt. Dies ist mit besonderen methodischen Schwierigkeiten verbunden, welche die Autoren im Supplement zu ihrer Arbeit ausführlich diskutieren. So muss z. B. sichergestellt sein, dass Begriffe wie „like“ („mögen“) nicht mit dem Begriff „like“ (im Sinne von „ist ähnlich“) in einen Topf geworfen werden. Auch die Frage nach der

11 "For example, Youn and colleagues conducted one particularly insightful analysis in which they showed that 22 Swadesh list concepts- which are theorized to be universally expressed and understood-had similar colexification patterns around the world based on the structure of colexification communities" [10]. “[...] languages were more likely to colexify concepts such as "water" and "sea," than concepts such as "sun" and "water," implying that speakers of these languages viewed "water" and "sea" as semantically similar concepts and "sun" and "water" as distinct. We use a similar approach to estimate the variation and structure of emotion semantics across language families" [10].
Repräsentativität der Daten (häufige Sprachen haben viele Wörter für viele Begriffe und stammen vor allem aus Europa, Asien und Amerika) wird eingehend diskutiert. Die Netzwerke wurden zunächst mit allen Begriffen (also auch mit der großen Zahl der Nichtemotionsbegriffe) berechnet, um bei den Kolexifikationen nicht solche zu übersehen, die über Nichtemotionsbegriffe verlaufen. Mit den Worten der Autoren: „Der Beginn mit einem solchen [allumfassenden] Netzwerk erlaubte die Schätzung der Kolexifikation von Emotionsbegriffen sowohl mit anderen Emotionsbegriffen als auch mit Nichtemotionsbegriffen, was eine wesentlich bessere Übersicht der semantischen Struktur der Emotionsbegriffe vermittelt als die Kolexifikation nur von Emotionsbegriffen. So sind beispielsweise ,Wut' und ,Neid“ in mehreren Sprachen mit ,Streit' kolexifiziert, was Informationen zum Zusammenhang von ,Wut' und ,Neid“ liefert, die über die [direkte] Kolexifikation der beiden Begriffe hinausgeht“12 [10].

Das allumfassende Netzwerk wurde dann mit einem besonderen Verfahren - der Berechnung von random walk probabilities - weiter ausgewertet. Man kann sich dieses Verfahren so vorstellen, dass der Computer per Zufall von einem Knoten im Netz zu einem anderen hüpft und die Anzahl der Schritte berechnet, die man braucht, um von einem bestimmten Knoten zu einem anderen bestimmten Knoten zu gelangen. Die durchschnittliche Anzahl der Schritte verhält sich umgekehrt zur Wahrscheinlichkeit, bei einem solchen random walk durch das Netzwerk auf den anderen Begriff zu gelangen. Um bei dem gerade verwendeten Beispiel zu bleiben: „Sofern ,Wut‘ und ,Neid“ miteinender kolixifiziert sind, und ebenso mit vielen weiteren jeweils gleichen Begriffen, haben sie

12 "Beginning with this network allowed us to estimate with a high degree of accuracy how emotion concepts were colexified with both emotion concepts and non-emotion concepts, which gives a more complete impression of the semantic structure of an emotion term than emotion concept to emotion concept colexifications alone. For example, both "anger" and "envy" are colexified in several languages with the concept "quarrel," and this offers information about the relationship between "anger" and "envy" above and beyond the direct colexification between these emotion concepts."
- Tab. 1 Liste der 24 Emotionen, in der Reihenfolge des Auftretens in den insgesamt 2474 Sprachen (nach Daten aus [10]).

\begin{tabular}{|c|c|c|}
\hline Emotion & Languages & $\begin{array}{l}\text { Colexifi- } \\
\text { cations }\end{array}$ \\
\hline Good & 2426 & 650 \\
\hline Bad & 1786 & 417 \\
\hline Want & 739 & 1008 \\
\hline Love & 712 & 315 \\
\hline Hate & 602 & 111 \\
\hline Happy & 570 & 152 \\
\hline Grief & 562 & 156 \\
\hline Shame & 475 & 45 \\
\hline Fear & 533 & 156 \\
\hline Anger & 353 & 112 \\
\hline Envy & 346 & 48 \\
\hline Proud & 335 & 50 \\
\hline Regret & 328 & 75 \\
\hline Anxiety & 321 & 68 \\
\hline Pity & 310 & 45 \\
\hline Surprised & 308 & 26 \\
\hline Hope & 297 & 113 \\
\hline Like & 245 & 109 \\
\hline Sad & 167 & 15 \\
\hline Merry & 118 & 9 \\
\hline Joy & 102 & 6 \\
\hline Desire & 58 & 89 \\
\hline Gloomy & 16 & 1 \\
\hline Worry & 8 & 4 \\
\hline
\end{tabular}

eine höhere Random-walk-Wahrscheinlichkeit. Wären sie andererseits nicht kolexifiziert und hätten sie zudem unterschiedliche Mengen von anderen Begriffen, mit denen sie kolexifiziert wären, würden ihre Random-walk-Wahrscheinlichkeiten gegen Null gehen"13 [10]. Die Anzahl der Kolexifikationen im Datensatz betrug 66140 , die 24 Emotionen sind in > Tab. 1 wiedergegeben. Aus der Wahrscheinlichkeitsmatrix der Kolexifikation im Netzwerk aller Begriffe wurde das Sub-Netzwerk erstellt, das nur

13 "[...] if "anger" and "envy" were colexified with each other, and were also colexified with many of the same concepts, they would have higher random walk probabilities. On the other hand, if they were not colexified with each other and had different sets of colexified concepts, their random walk probabilities would approach 0 ." 


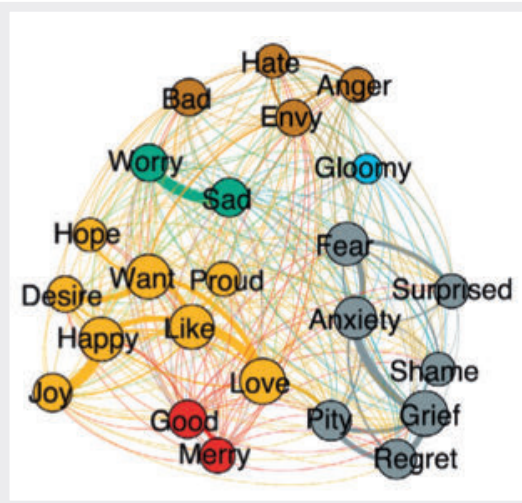

- Abb. 5 Kolexifikations-Netzwerk über alle untersuchten Sprachen hinweg [nach Daten aus [10]). Begriffe („Knoten“), die zum gleichen Cluster gehören, sind in gleicher Farbe dargestellt, die Dicke der Verbindungen („Kanten“) repräsentiert das Ausmaß der Kolexifikation.

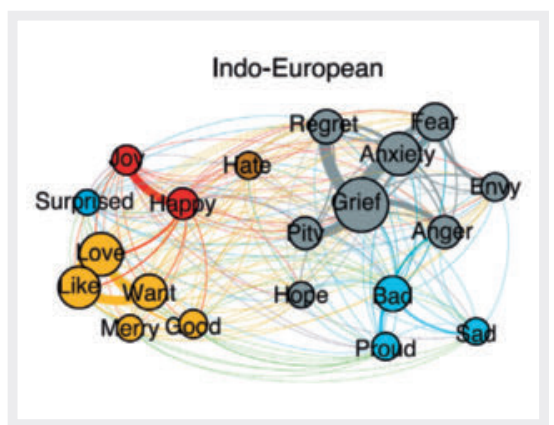

- Abb. 6 Kolexifikations-Netzwerk für die indo-europäische Sprachfamilie (nach Daten aus [10]).

aus diesen 24 Emotionen gebildet wird, das den Grad ihrer Kolexifikation angibt.

Ganz prinzipiell funktioniert Kolexifikation nur zwischen mehreren Sprachen (man braucht ja Übersetzungen!). Daher kann man mit dieser Methode auch nicht die semantischen Netzwerke einzelner Sprachen untersuchen oder gar vergleichen. Auf der Ebene von Sprachfamilien hingegen funktioniert das Verfahren, weswegen die gerade beschriebene Prozedur innerhalb der (im untersuchten Korpus enthaltenen) 33 Sprachfamilien mit jeweils mehr als 10 Sprachen durchgeführt wurde, um sprachabhängige Unterschiede zwischen den Familien zu identifizieren. Zur weiteren Ordnung und Aufarbeitung der Ergebnisse wurde ferner ein Optimierungsverfahren (genannt „Cluster Optimal community
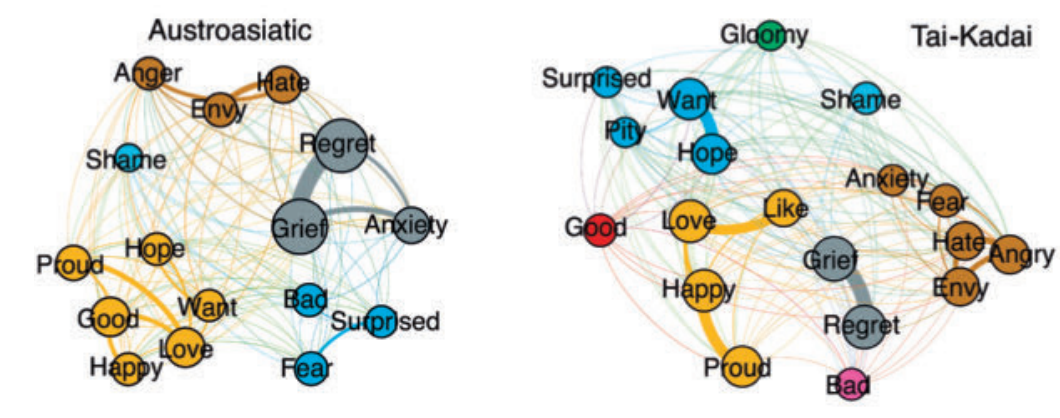

- Abb. 7 Kolexifikations-Netzwerke der austro-asiatischen Sprachfamilie (links) und der Sprachfamilie Tai-Kadai (nach Daten aus [10]).

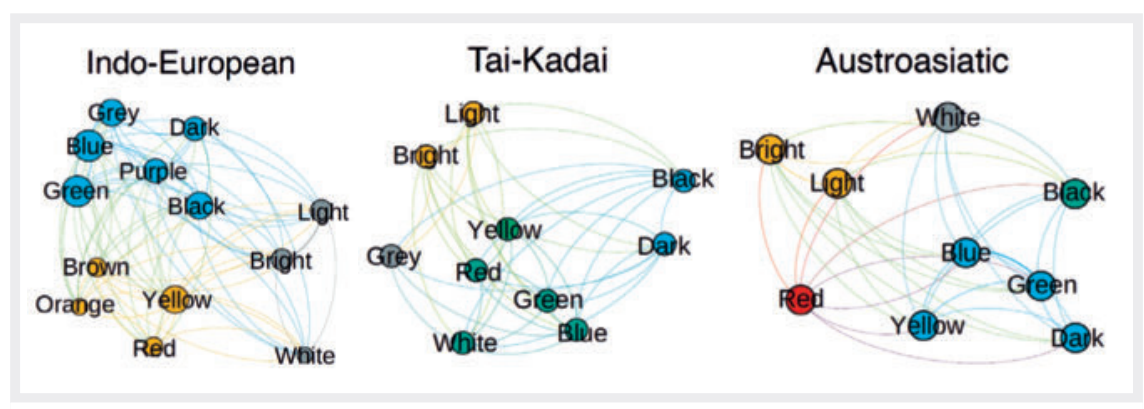

- Abb. 8 Aus 13 Farbwörtern durch Kolexifikation „destillierte“ Farbnetzwerke, die zeigen mögen, dass auch Farben, die nach allgemeiner Auffassung deutlich "natürlicher“ sind als Emotionen (und weniger kulturell determiniert), jedoch keineswegs „universell“ sind (nach Daten aus [10])

detection algorithm“14) verwendet, das die Emotionsbegriffe nach Ähnlichkeit („,communities“) gruppiert und ferner die grafische Darstellung der Netzwerke erlaubt. In - Abb. $\mathbf{5}$ ist die Struktur des allumfassenden Netzwerks dargestellt. \ Abb. 6 zeigt das Kolexikationsnetzwerk für die Indo-Europäische Sprachfamilie, $>$ Abb. 7 zeigt entsprechende Netzwerke für 2 weitere Sprachfamilien.

Die für einzelne Sprachfamilien berechneten Netzwerke weisen auf deutliche Unterschiede in den Bedeutungshöfen (ange-

14 Dieses sehr rechenintensive Verfahren liefert immer ein Struktur-Optimum; mit den Worten der Autoren: „Whereas most modularity-based community detection approaches use heuristics to identify local optima, Cluster Optimal [so der Name der Prozedur] finds the global maximum. That is, it will always detect the mathematically optimal community structure in the sense of modularity maximization. Cluster Optimal is not used more widely to detect community structure in networks because it is so computationally intensive“ [10]. zeigt durch Unterschiede in deren „Nebenbedeutungen“, die sich in Unterschieden der Kolexifikation zeigen) der Emotionsbegriffe hin. Die Autoren geben auch statistische Maße für die Strukturunterschiede der Netzwerke (z. B. , adjusted Rand indices, ARIs“) an, die zwischen -1 (sehr unähnlich) und 1 (sehr ähnlich) variieren können, wobei „0“ „völlig zufällig“ bedeutet. Weil diese Maße weitgehend unbekannt sind und ihre Interpretation damit schwer nachvollziehbar ist, verwenden sie einen aus meiner Sicht cleveren Vergleich: Sie führten die gesamte Analyse nochmals durch, konzentrierten sich jedoch dabei nicht auf 24 Emotionen, die in der ursprünglichen Begriffsliste angeführt waren, sondern auf 13 Farben ( $\triangleright$ Abb. 8).

Bekanntermaßen wird die Semantik kontrovers diskutiert, angefangen mit der heute widerlegten Behauptung, dass die „Eskimos“ (Inuit) 15 Wörter für „Schnee“ verwenden würden (es sind tatsächlich nur 2) über das Belächeln dieser Beispiele in den 1980er-Jahren bis hin zu neuen 
empirischen Daten, die tatsächlich deutliche kulturelle Unterschiede in der Anzahl und Bedeutung von Farbwörtern anzeigen $[4,7,13,28]$. Neuronale Netzwerk-Modelle $[21,25,26]$ sowie Untersuchungen mit elektrophysiologischen [16] oder funktionell-bildgebenden Verfahren [17] zeigen sehr deutlich, dass und wie hochstufige Repräsentationen Einfluss auf einfache, rasche, „automatische“ und unbewusst ablaufende Prozesse der Wahrnehmung haben können.

Die in $\mathbf{A} \mathbf{A b} \mathbf{b} . \mathbf{8}$ dargestellten Netzwerke der Bedeutung von Farbwörtern zeigen deutlich, dass es hier sprachbezogene, also kulturelle, Unterschiede gibt. Werden diese mit der gleichen Methodik näher analysiert (Stichwort: ARIs), so zeigt sich den Autoren zufolge bei den Emotionen eine höhere kulturelle Variation als bei den Farben. Weitere Analysen zeigten, dass die geografische Distanz der untersuchten Sprachen sich auf die Ähnlichkeit der Semantik der Emotionsbegriffe auswirkt, was auf kulturellen Austausch und damit ganz allgemein auf kulturelle Determiniertheit schließen lasse. Weitere Experimente konnten zeigen, dass aber auch die Biologie der Emotionen, nämlich deren Grad der Erregung (arousal) und deren Bewertung (valence), einen Einfluss auf die allgemeine semantische Struktur von Bedeutungsnetzwerken hat. Diese Struktur ist also nicht vollkommen kulturell determiniert und damit nicht beliebig (wie beispielsweise Automarken).

Aus meiner Sicht stellen die vorgestellten Arbeiten einen interessanten Anfang für weitere Forschungen dar. Daten gibt es heute sehr viele, auch Datensätze und Zusammenfassungen von Datensätzen. Es liegt an der Kreativität der Forscher, diese Daten mittels immer neuer Strategien auszuwerten und damit fruchtbar zu machen für ein tieferes Verständnis unserer selbst, unserer Kultur und unserer Biologie. Es gibt noch viel mehr auf der Welt als Farben und Emotionen!

\section{Interessenkonflikt}

Es liegt kein Interessenkonflikt vor.
Korrespondenzadresse

Prof. Dr. Dr. Manfred Spitzer

Universität Ulm

Abteilung für Psychiatrie

Leimgrubenweg 12-14

89075 Ulm

\section{Literatur}

[1] Brain CK, Sillent A. Evidence from the Swartkrans cave for the earliest use of fire. Nature 1988, 336: 464-466

[2] Bußmann H. Lexikon der Sprachwissenschaft. 4. Auflage. Stuttgart: Kröner; 2008

[3] Cibelli E, Xu Y, Austerweil JL, et al. The Sapir-Whorf Hypothesis and Probabilistic Inference: Evidence from the Domain of Color. PLoS ONE 2016; 11: e0158725.

[4] Davidoff J, Davies I, Roberson D. Colour categories in a stone-age tribe. Nature 1999; 398: 203-204

[5] González-Forero M, Gardner A. Inference of ecological and social drivers of human brain-size evolution. Nature 2018; 557: 554-557

[6] Gowlett JAJ, Wrangham RW. Earliest fire in Africa: towards the convergence of archaeological evidence and the cooking hypothesis. Azania: Archaeological Research in Africa 2013; 48: 5-30

[7] He H, Li J, Xiao Q, jiang S, et al. Language and Color Perception: Evidence From Mongolian and Chinese Speakers. Front Psychol 2019; 10: 551

[8] Henrich J. The secret of our success. Princeton: University Press; 2016

[9] Hua X, Greenhill SJ, Cardillo M, et al. The ecological drivers of variation in global language diversity. Nat Commun 2019; 10: 2047. doi: 10.1038/s41467-019-09842-2

[10] Jackson JC, Watts J, Henry TR, et al. Emotion semantics show both cultural variation and universal structure. Science 2019; 366 : 1517-1522

[11] Kay P, Cook RS. World Color Survey. In: Luo MR (Hrsg.). Encyclopedia of Color Science and Technology. New York: Springer; 2016

[12] Lindquist KA, Wager TD, Kober $\mathrm{H}$, et al. The brain basis of emotion: a meta-analytic review. Behav Brain Sci 2012; 35: 121-143

[13] Maier M, Abdel Rahman R. No matter how: Top-down effects of verbal and semantic category knowledge on early visual perception. Cogn Affect Behav Neurosci 2019; 19: 859-876

[14] Muthukrishna M, Doebeli M, Chudek M, et al. The Cultural Brain Hypothesis: How culture drives brain expansion, sociality, and life history. PLoS Comput Biol 2018; 14 : e1006504
[15] Pagel M, Meade A. The deep history of the number words. Phil Trans R Soc B 2017; 373: 20160517

[16] Pérez-Gay Juárez F, Sicotte T, Thériault C, et al. Category learning can alter perception and its neural correlates. PLoS ONE 2019; 14: e0226000

[17] Satpute AB, Nook EC, Narayanan S, et al. Emotions in "Black and White" or Shades of Gray? How We Think About Emotion Shapes Our Perception and Neural Representation of Emotion. Psychological Science 2019; 27: 1428-1442

[18] Skelton AE, Catchpole G, Abbott JT, et al. Biological origins of color categorization. PNAS 2017; 114: 5545-5550

[19] Spitzer M. Blaue Augen, blaues Meer. Nervenheilkunde 2017; 36: 839-844

[20] Spitzer M. Christiania, Science und Cocktails. Nervenheilkunde 2019; 38: 453-458

[21] Spitzer M. Erderwärmung und Vorurteile (Editorial). Nervenheilkunde 2020; 39: 6-9

[22] Spitzer M. Automatik im Kopf. Wie das Unbewusste arbeitet. In: Spitzer M, Bertram W (Hrsg.). Hirngespinste. Stuttgart: Schattauer; 2020 im Druck

[23] Swadesh M. Lexico-statistical dating of prehistoric ethnic contacts. Proc Am Philos Soc 1952; 96: 452-463

[24] Swadesh M. The origin and diversification of language. Edited post mortem by Joel Sherzer. Chicago, IL: Aldine, 1971

[25] Thielscher A, Kölle M, Neumann H, et al. Texture segmentation in human perception: A combined modeling and fMRI study. Neuroscience 2009; 151: 730-736

[26] Tomasello R, Garagnani M, Wennekers T, et al. Brain connections of words, perceptions and actions: A neurobiological model of spatio-temporal semantic activation in the human cortex. Neuropsychologia 2017; 98 : 111-129

[27] Whiten A, Ayala FJ, Feldman MW, et al. The extension of biology through culture. PNAS 2017; 114: 7775-7781

[28] Witzel C, Gegenfurtner KR. Categorical facilitation with equally discriminable colors. Journal of Vision 2015; 15: 1-33

[29] Youn H, Suttond L, Smith E, et al. On the universal structure of human lexical semantics. PNAS 2016; 113: 1766-1771

\section{Bibliografie}

DOI https://doi.org/10.1055/a-0976-0596

Nervenheilkunde 2020; 39: 75-81

(c) Georg Thieme Verlag KG Stuttgart . New York ISSN 0722-1541 